\title{
ROBHAND, UN EXOESQUELETO DE MANO PARA LA REHABILITACIÓN NEUROMOTORA APLICANDO TERAPIAS ACTIVAS Y PASIVAS
}

\author{
Ana Cisnal, Víctor Lobo, Víctor Moreno, Juan C. Fraile \\ ITAP - Instituto de las Tecnologías Avanzadas de la Producción, Universidad de Valladolid. \\ cisnal@ieee.org ; \{ victor.lobo, victor.moreno\}@alumnos.uva.es ; jcfraile@eii.uva.es \\ Rubén Alonso, Javier P. Turiel \\ Área de Bienestar y Salud, Centro Tecnológico Fundación Cartif \\ \{rubalo, javper\}@cartif.es
}

\begin{abstract}
Resumen
Presentamos el planteamiento y estado actual de ejecución de un proyecto que se está realizando mediante la colaboración de una empresa (CyL Imas D, Salamanca), dos centros asistenciales (Hospital Clínico Universitario de Valladolid y Corporación de Rehabilitación Club de Leones Cruz del Sur, Chile) y un centro de investigación vinculado a una Universidad (Instituto Universitario ITAP, de la Universidad de Valladolid). El objetivo principal de este proyecto es la creación, desarrollo, implantación y pruebas clínicas de un nuevo prototipo de robot háptico activo para mantenimiento y rehabilitación neuromotora de la mano en pacientes con daño cerebral adquirido. Se describe el planteamiento del proyecto, se hace una descripción técnica de los principales componentes del sistema y se resume su estado de ejecución.
\end{abstract}

Palabras clave: Robótica asistencial, Exoesqueletos, Neurorehabilitación, Control háptico, sEMG

\section{INTRODUCCIÓN}

Las enfermedades cerebrovasculares constituyen, en la actualidad, uno de los más importantes problemas de salud pública. Son la tercera causa de muerte en el mundo occidental, la primera causa de invalidez permanente entre las personas adultas y una de las principales causas de déficit neurológico en el anciano. Entre las personas que sufren un Accidente Cerebro-Vascular (ACV), aproximadamente un 75\% sufren paresia de las extremidades superiores [15]. Seis meses después del accidente cerebrovascular, el $65 \%$ no se recuperan por completo. Además, el déficit crónico a menudo se puede encontrar en el nivel de la mano [2], y la probabilidad de recuperar el uso funcional de la mano deteriorada ha sido descrita como "más bien baja" [10].

La utilización de robots para rehabilitación de la mano está suponiendo una redefinición de las técnicas de rehabilitación actuales [5]. Estos robots pueden controlar con precisión la fuerza aplicada (ayuda al paciente) durante la realización de la terapia, y adaptar progresivamente la asistencia y/o resistencia a las habilidades de los pacientes. Mientras que la rehabilitación clásica está limitada por la observación subjetiva de los terapeutas y los pacientes, la utilización de dispositivos robóticos para rehabilitación permite cuantificar con precisión los progresos realizados por los pacientes. Además, los tratamientos pueden ser diseñados para adaptarse al estado del paciente [6], [7].

En esta comunicación presentamos el estado actual de desarrollo de Robhand, un nuevo robot para rehabilitación de mano, mediante terapias activas y pasivas, para pacientes que tienen discapacidad neuromotora debido a un ACV. El entorno de rehabilitación integra un exoesqueleto de mano, un sistema de registro y procesamiento de señales electromiográficas (sEMG) y un entorno software para la gestión de pacientes, terapias y la realización de tareas de rehabilitación basadas en entornos virtuales. Su desarrollo se enmarca en un proyecto de colaboración entre una empresa (CyL Imas D) y un centro de investigación vinculado a una Universidad (Instituto Universitario ITAP, de la Universidad de Valladolid), con la colaboración de centros asistenciales (Hospital Clínico Universitario de Valladolid y Corporación de Rehabilitación Club de Leones Cruz del Sur, Chile), y es un ejemplo de los resultados que puede dar la colaboración públicoprivada en el ámbito de la I+D+i.

\section{ELEMENTOS DEL SISTEMA ROBHAND}

El componente base de Robhand es un dispositivo electromecánico de tipo exoesqueleto, que se fija a la mano del paciente y proporciona asistencia para la realización de diferentes tipos de terapias de rehabilitación del movimiento de los dedos. 
Puesto que la finalidad del diseño es la rehabilitación de la mano, el dispositivo debe tener en cuenta necesidades y requisitos específicos de esta. En su diseño se tienen en cuenta los datos de diferentes parámetros antropométricos de la mano, a partir de los cuales, y de otros requisitos y restricciones, se ha elaborado un modelo cinemático que se presenta en el apartado correspondiente.

En cuanto al control del funcionamiento del dispositivo, la estrategia que hemos planteado busca fundamentalmente lograr dos objetivos: implementar en nuestro exoesqueleto de mano el paradigma "Assist as Needed" (asistencia solo cuando es necesario y con la intensidad adecuada) y la detección de la "intención de movimiento" del paciente, midiendo señales de electromiografía (sEMG) de los músculos del brazo-antebrazo implicados en el movimiento de sus dedos.

El sistema se completa con un entorno software que facilita al personal clínico la gestión de pacientes y terapias, y proporciona una plataforma de ayuda a la ejecución de tareas de rehabilitación, mediante diferentes tipos de entornos virtuales con los que el paciente puede interactuar.

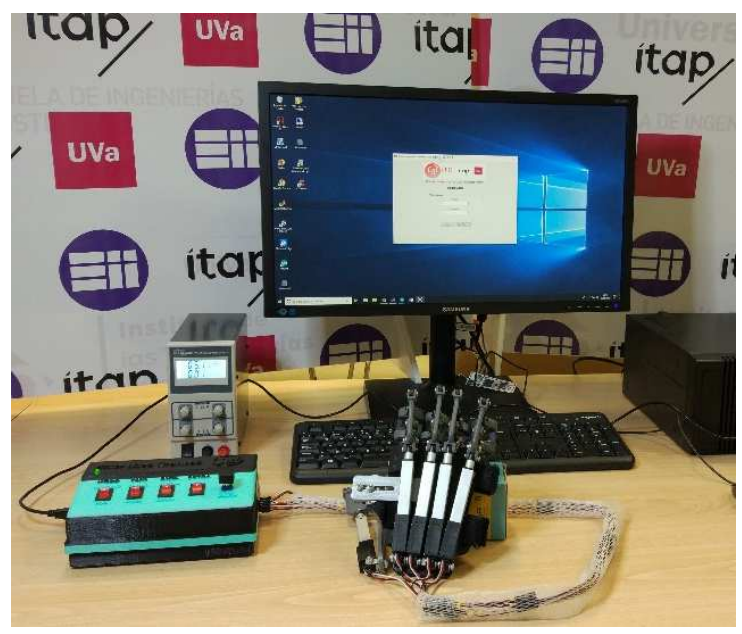

Figura 1: Componentes del sistema Robhand para rehabilitación de mano.

\section{ASPECTOS DE DISEÑO}

En su versión de desarrollo actual, el dispositivo RobHand, con un peso total de 285 gramos, incorpora mecanismos que permiten la flexión y extensión de la falange metacarpofalángica (MCP) y de la falange interfalángica proximal (PIP) de cada dedo, mediante cinco servomotores lineales L2-50210-6-R (Firgelli) controlados mediante un Arduino ATMega 2650, que se comunica a una aplicación desarrollada para la realización de terapias pasivas en el ordenador.

\subsection{DISEÑO MECÁNICO}

La mano humana puede modelarse de forma simplificada como un mecanismo (fig. 2), que consta de tres grados de libertad en los dedos índice, corazón, anular y meñique; y dos grados de libertad para el dedo pulgar.

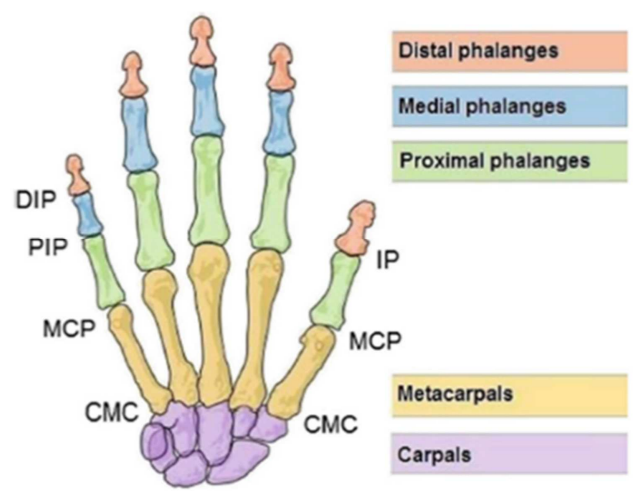

Figura 2: Articulaciones y huesos de la mano derecha (según Andrianesis y Tzes [1]).

Para simplificar el mecanismo y conseguir un reducido tamaño y peso, cada dedo del exoesqueleto se diseña con dos de grados de libertad correspondientes a las articulaciones MCP y PIP, a excepción del pulgar que cuenta sólo con un grado de libertad (MCP). Las dimensiones del exoesqueleto se han diseñado acorde al estudio antropomórfico del mano realizado en [17] utilizando los valores promedios de la muestra de estudio, teniendo como objetivo en un futuro el desarrollo de piezas intercambiables para diferentes tamaños de mano.

La estructura mecánica del sistema (fig. 3) se compone de cinco subconjuntos, uno para cada dedo, formados por ocho piezas, además de la tornillería que los une. Cada subconjunto se monta directamente sobre una placa base, a excepción del pulgar el cual, debido a que se encuentra en oposición al resto de dedos, precisa de más piezas que nos permita colocarlo en una disposición correcta.

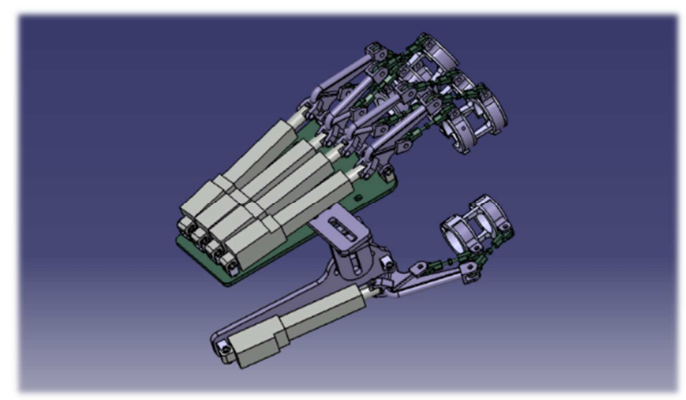

Figura 3: Diseño del exoesqueleto de mano en CATIA v5. 
Cada subconjunto (fig. 4) cuenta con una pieza intermedia y dos tirantes que se encargan de transmitir la fuerza del motor hacia los anillos, estando estos últimos en contacto con los dedos.

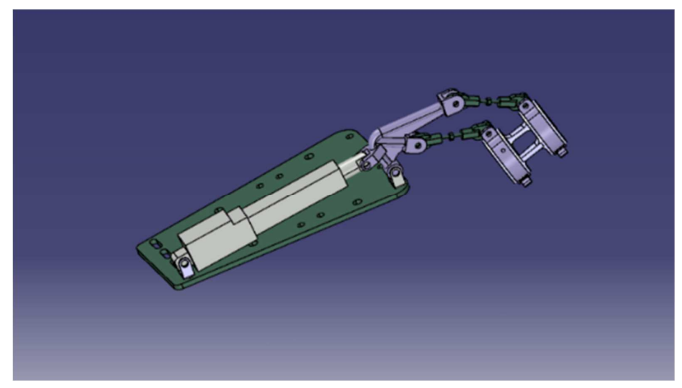

Figura 4: Diseño del dedo índice en CATIA V56R2016.

Las piezas no comerciales están fabricadas mediante impresión 3D en material que les permite soportar los esfuerzos a los que se ven sometidas (PLA); además las piezas que se encuentran en contacto con los dedos son de material flexible (Filaflex 82a), permitiendo así una mayor adaptabilidad a los distintos tamaños de dedos, y evitando que provoquen algún tipo de lesión o herida.

\subsection{CINEMÁTICA DIRECTA}

El estudio cinemático directo del sistema tiene como objetivo la determinación del ángulo y velocidad angular de las articulaciones MCP y PIP de cada dedo en función de la longitud del vástago del motor. En la figura 5 se muestra la parametrización de los ángulos y longitudes del sistema, tomando el eje de rotación de la articulación MCP como referencia, ya que este puede considerarse fijo [11]:
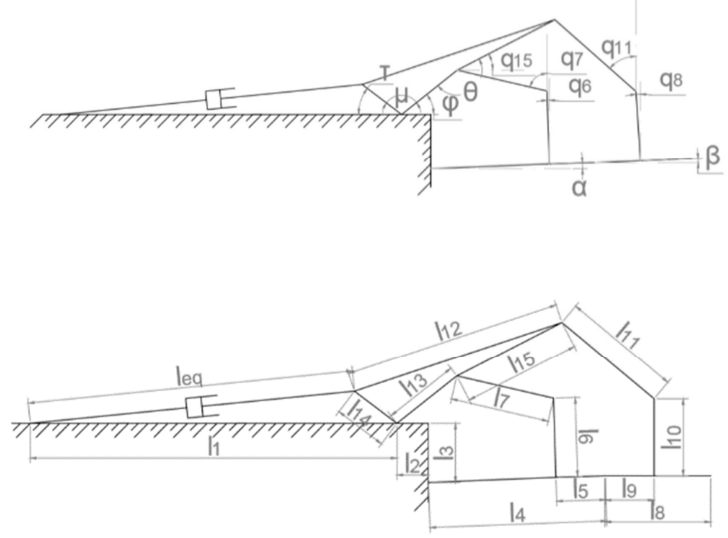

Figura 5: Parametrización de los ángulos y las longitudes en Autocad 2D.

Mediante el método geométrico de los anillos, se relacionan las diferentes variables geométricas que caracterizan el mecanismo, obteniendo un sistema de ecuaciones para cada una de las falanges:
- $\quad$ Falange proximal; las incógnitas son $\alpha$ y $q_{7}$ :

$\left\{\begin{array}{c}\bar{l}_{2}+\left(\bar{l}_{4}-\bar{l}_{5}\right) \cos \alpha+\bar{l}_{6} \sin q_{6}+\bar{l}_{7} \sin q_{7}-\bar{l}_{13} \cos \varphi=0 \\ -\bar{l}_{3}+\left(\bar{l}_{4}-\bar{l}_{5}\right) \sin \alpha+\bar{l}_{6} \cos q_{6}+\bar{l}_{7} \cos q_{7}-\bar{l}_{13} \sin \varphi=0\end{array}\right.$

- $\quad$ Falange distal, siendo las incógnitas $\beta$ y $q_{11}$ :

$$
\begin{aligned}
& \bar{l}_{2}+\bar{l}_{4} \mathrm{C}(\alpha)+\bar{l}_{9} \mathrm{C}(\beta)-\bar{l}_{10} \mathrm{~S}\left(q_{8}\right)+ \\
& \quad+\bar{l}_{11} \mathrm{~S}\left(q_{11}\right)-\bar{l}_{15} \mathrm{C}\left(q_{15}\right)-\bar{l}_{13} \mathrm{C}(\varphi)=0 \\
& -\bar{l}_{3}+\bar{l}_{4} \mathrm{~S}(\alpha)+\bar{l}_{9} \mathrm{~S}(\beta)+\bar{l}_{10} \mathrm{C}\left(q_{8}\right)+ \\
& \quad+\bar{l}_{11} \mathrm{C}\left(q_{11}\right)-\bar{l}_{15} \mathrm{~S}\left(q_{15}\right)-\bar{l}_{13} \mathrm{~S}(\varphi)=0
\end{aligned}
$$

Una vez determinadas estas cuatro incógnitas, se procede al cálculo de la velocidad angular de las articulaciones $\operatorname{MCP}(\dot{\alpha})$ y $\operatorname{PIP}(\dot{\beta})$ :

$$
\begin{aligned}
& \dot{\alpha}=\frac{\dot{\varphi}}{l_{45} \mathrm{C}\left(\alpha-q_{7}\right)}\left(l_{13} \mathrm{~S}\left(q_{7}\right) \mathrm{S}(\varphi)+l_{13} \mathrm{C}\left(q_{7}\right) \mathrm{C}(\varphi)\right) \\
& \dot{\beta}=\frac{\dot{\varphi}}{l_{9} \mathrm{C}\left(\beta-q_{11}\right)}\left(l_{13} \mathrm{~S}\left(q_{11}\right) \mathrm{S}(\varphi)+l_{13} \mathrm{C}\left(q_{11}\right) \mathrm{C}(\varphi)\right)
\end{aligned}
$$

A partir del estudio cinemático directo, también se determina la posición de las articulaciones interfalángicas proximal (PIP) y distal (DIP) tomando como origen de coordenadas la articulación MCP:

$$
\begin{gathered}
\left\{\begin{array}{l}
x_{P I P}=l_{4} \cos \alpha \\
y_{P I P}=l_{4} \sin \alpha
\end{array}\right. \\
\left\{\begin{array}{l}
x_{D I P}=l_{4} \cos \alpha+l_{8} \cos \beta \\
y_{D I P}=l_{4} \sin \alpha+l_{8} \sin \beta
\end{array}\right.
\end{gathered}
$$

\section{CONTROL DEL DISPOSITIVO}

Los actuadores, que determinan la trayectoria de cada mecanismo simple o subconjunto del exoesqueleto, son controlados por un sistema de control háptico. Sin embargo, para la implementación de este tipo de control se debe conocer de manera previa el movimiento a asistir, por lo que se propone la integración de una estrategia para detectar la intencionalidad del paciente, mediante el procesamiento de señales sEMG

\subsection{ARQUITECTURA DE CONTROL}

La estrategia de control planteada busca poder implementar el paradigma "Assist as Needed" y la detección de la "intención de movimiento" del paciente, midiendo señales de electromiografía (sEMG) de los músculos del brazo-antebrazo del paciente implicados en el movimiento de sus dedos. Desde el punto de vista de la implementación del modelo conceptual planteado, la arquitectura diseñada para el control del exoesqueleto de mano se muestra en la figura 6. 
La capa L-1 constituye el nivel de control superior. Esta capa de control recibe información del estado del exoesqueleto de la capa intermedia de control L2, y envía las consignas al nivel de control L-2. L-2 constituye el nivel intermedio de control. Esta capa de control intermedio recibe las consignas de la capa L-1 y envía las consignas al nivel inferior de control L-1. El objetivo de esta capa L-2 es conseguir que el robot de rehabilitación se comporte como un sistema con impedancia mecánica igual al requerido por la capa superior L-1.

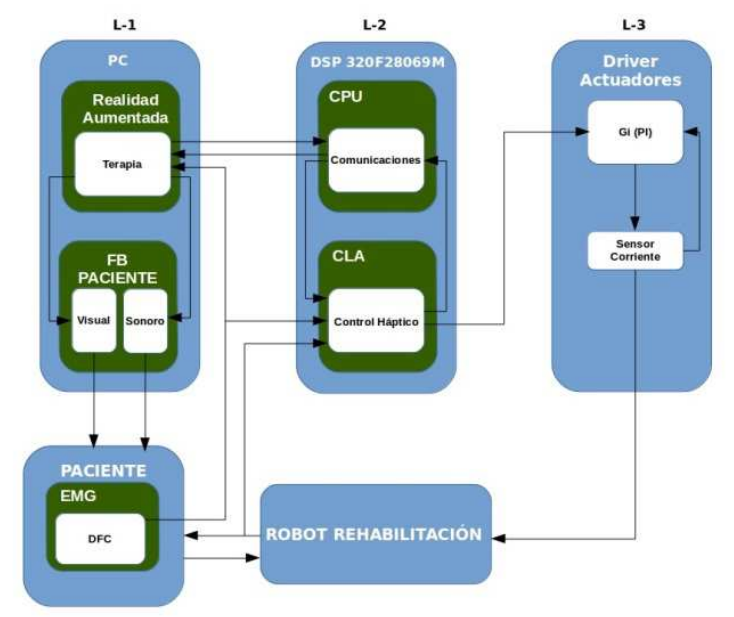

Figura 6: Diagrama de bloques de los 3 niveles de la arquitectura de control de Robhand.

L-3 constituye el nivel inferior de control. Esta capa de control inferior recibe las consignas de la capa intermedia L-2 y controla los actuadores del exoesqueleto de rehabilitación. El sistema de control es háptico, implementado a través de un algoritmo de admitancia, en vez de un algoritmo de impedancia, debido a que los actuadores no son reversibles al tener una reducción muy elevada (200:1). Este control se ha implementado en un microcontrolador ATMEGA 2650 y se ha programado con el IDE de Arduino.

El diagrama de bloques del sistema de control de un servomotor (fig. 7), está definido por los siguientes parámetros:

- $\quad$ o indica la posición, y los sufijos d y m indican motor y deseada.

- $\quad$ F indica fuerza y los sufijos m, p y r indican motor, paciente y resultante.

- $\quad$ e es la señal de error, I es la corriente en el motor $n, K_{i}$ es la constante de fuerza del actuador y $\mathrm{U}$ es la tensión aplicada al motor.

La tarjeta Arduino mide las fuerzas que el usuario efectúa sobre el exoesqueleto, y reacciona con un movimiento (posición, velocidad, aceleración). Se controla el movimiento de cada uno de los actuadores. Con lo cual, el sistema actúa como una admitancia y el operador como una impedancia. El sistema RobHand actúa como una "fuente de velocidad".

En la figura se representa en enfoque que estamos desarrollando en la actualidad, que incluye al paciente dentro del lazo de control con el fin de determinar el grado de asistencia proporcionado por el exoesqueleto en función de la intencionalidad de movimiento del paciente. Para ello, se incluye la estrategia 'Direct Force Control' (DFC) al actual sistema de control (fig. 8), consiguiendo de esta manera, una asistencia ponderada entre el control háptico y el DFC.

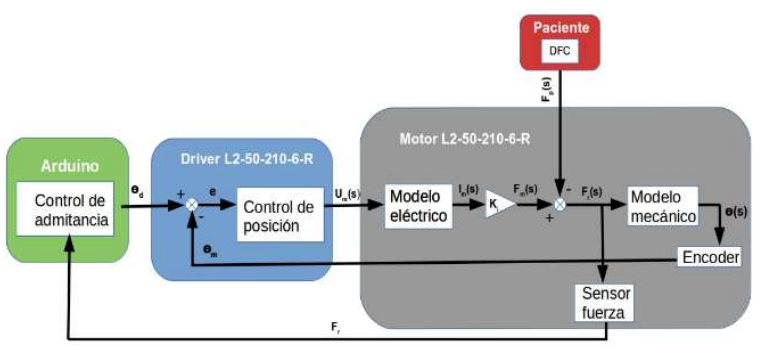

Figura 7: Diagrama de control planteado, que incluye al paciente.

\subsection{DETECCIÓN DE INTENCIONALIDAD DEL MOVIMIENTO}

Para detectar la intencionalidad de movimiento del usuario se propone la estrategia DFC [3], que consiste en calcular el par ejercicio en la articulación sobre la que actúa el robot, resultante de la activación muscular de las señales electromiográficas (sEMG) medidas en el brazo-antebrazo del paciente. Este par puede ser interpretado, como la contribución del paciente al movimiento de sus dedos, durante la realización de la terapia de rehabilitación.

Las primeras pruebas de adquisición de señales sEMG se realizaron mediante un equipo de adquisición de señales biomédicas comercial (MP150, BIOPAC) con una frecuencia de muestreo de $1000 \mathrm{~Hz}$. Los electrodos superficiales se sitúan en los músculos extensor común (ED) y flexor cubito del carpus (FU), que son dos de los músculos responsables del cierre y apertura de la mano, y el electrodo de referencia se coloca en el olecranon.

Se implementa el método umbral para la detección de activación muscular en Matlab 2016b (MathWorks). El método umbral determina que se ha producido una activación muscular si las señales EMG normalizadas superan un umbral [8], [12], [13]. En primer lugar, se realiza el filtrado de las señales mediante un filtro Notch, pasa bajo y pasa alto con una frecuencia de corte de 50, 10 y $500 \mathrm{~Hz}$, respectivamente. 
Posteriormente, se realiza la normalización de la señal utilizando una ventana móvil de $300 \mathrm{~ms}$, para ello se realiza un rectificado dinámico de la señal original respecto a su línea base y se calcula el porcentaje del valor cuadrático medio de la señal rectificada (RMS) respecto al valor de referencia de actividad submáximo (RVC).

Mediante el método umbral implementado se determina cuando se produce una activación muscular de los músculos ED y FU, encargados de la apertura y cierre de la mano, respectivamente (ver línea roja, fig. 8). Sin embargo, para considerar una apertura y cierre de la mano, la señal EMG normalizada tiene que superar el valor umbral una duración de tiempo mínima de $350 \mathrm{~ms}$ (ver línea discontinua negra, fig. 8). Este enfoque es similar al utilizado en [14]:

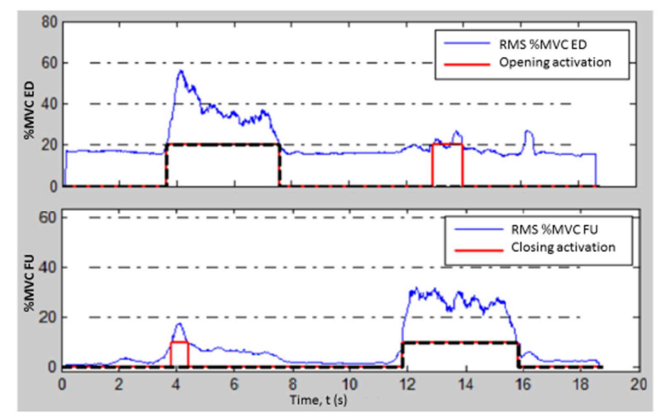

Figura 8: Detección de apertura y cierre de la mano.

El método umbral implementado tiene una tasa de acierto del 89.7 y $87.2 \%$ para la detección de apertura y cierre de la mano, respectivamente.

Tras esta primera etapa de implementación y verificación del método umbral para la detección de activación muscular, se procede al diseño y construcción de una placa para el pretratamiento de las señales sEMG (fig. 9), con el fin de reemplazar el sistema comercial utilizado anteriormente.

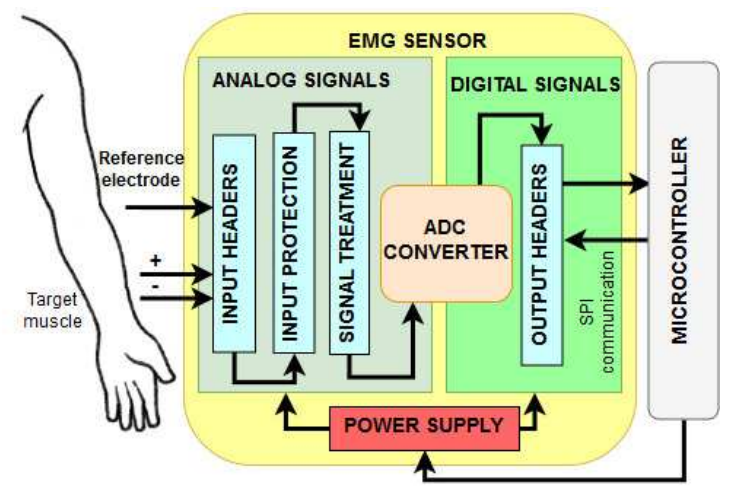

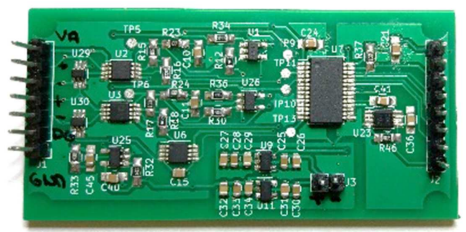

Figura 9: Plataforma hardware desarrollada para el pre-tratamiento analógico de las señales sEMG

En este momento, se está trabajando en la implementación de un método umbral-proporcional para la determinación de la fuerza producida por el músculo, ya que hay estudios que afirman que la fuerza de los músculos está relacionada de manera lineal con las señales sEMG [9]. La fuerza calculada por este método se utilizará como consigna de control para la estrategia DFC.

\section{ENTORNO SOFTWARE}

Se ha desarrollado un entorno software que proporciona soporte al terapeuta para la gestión de pacientes y terapias de rehabilitación pasivas que se realizan con la ayuda del exoesqueleto. Mediante esta aplicación, el terapeuta selecciona el paciente que realiza la sesión y define la terapia a realizar por el mismo, de acuerdo con su historial clínico y de terapias realizadas.

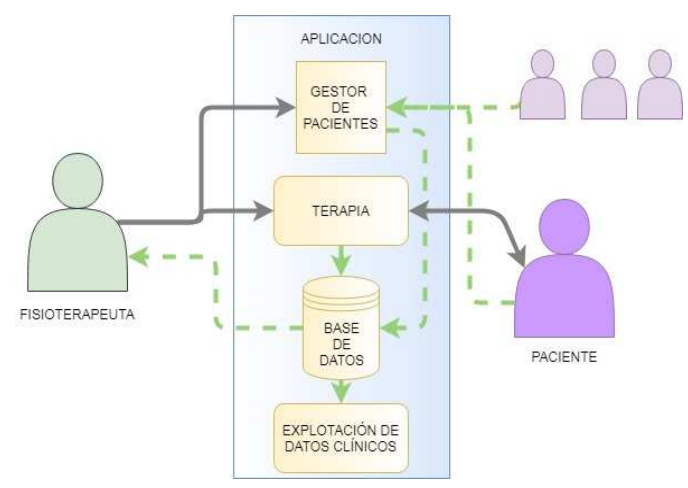

Figura 10: Diagrama de funcionalidad pacienteterapeuta del entorno software.

\subsection{GESTIÓN DE PACIENTES Y TERAPIAS}

El entorno de gestión de pacientes y terapias permite elegir el paciente de entre los registrados en la aplicación, o crear uno nuevo si no existe, revisar el historial y definir o configurar la sesión de terapia. Al iniciar la aplicación, aparece una pantalla (Fig. 11) en la que el fisioterapeuta introduce su usuario y contraseña. También permite crear nuevos perfiles de fisioterapeutas. 


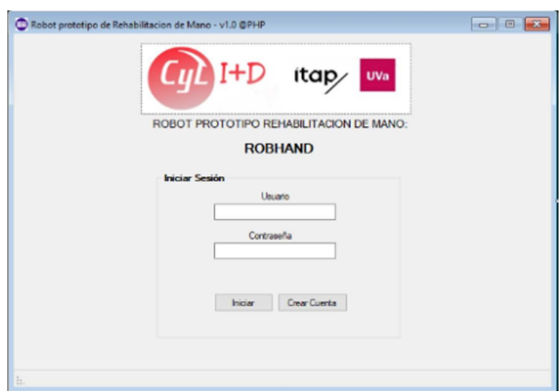

Figura 11: Pantalla de acceso a la aplicación

Una vez introducido correctamente el usuario y contraseña, emerge otra pantalla (Fig. 12, (a)) en la que se muestran los pacientes que tiene a cargo ese fisioterapeuta. Cada paciente tiene un registro con información relativa al número de terapias realizadas y el tipo de las mismas, el tiempo total de ejercicio y un registro de observaciones.

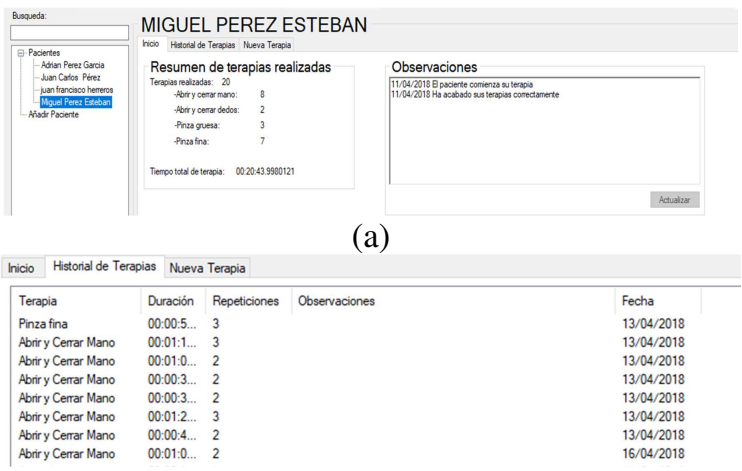

(b)

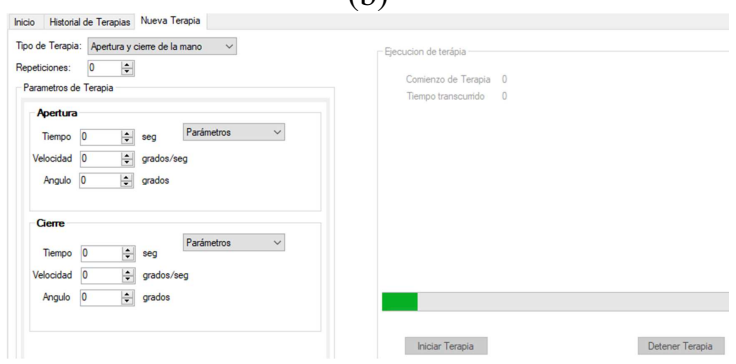

(c)

Figura 12: Capturas de pantalla mostrando diferentes funcionalidades de la aplicación

En la pestaña Historial de Terapias (fig. 12, (b)), se muestran, ordenadas por fecha, las terapias realizadas por el paciente seleccionado, con el número de repeticiones y la duración de cada una. Otra pantalla (Fig. 12, (c)) permite la elección de la terapia y la introducción de los parámetros necesarios para la caracterización de la terapia de acuerdo con las necesidades del paciente.

\subsection{TERAPIAS}

Los ejercicios de rehabilitación programados en entorno software son terapias convencionales, basadas en la repetición de movimientos que implican la flexión y extensión de los dedos, y son:
- Abrir y cerrar la mano: realiza la flexión y extensión de la mano completa

- Abrir y cerrar los dedos: realiza la flexión y extensión de los dedos de la mano de manera individual.

- Pinza fina y pinza gruesa: realiza la apertura y cierre del pulgar contra el dedo índice (pinza fina) o contra los cuatro dedos de la mano (pinza gruesa).

El terapeuta puede adecuar las terapias anteriormente descritas para cada paciente, ya que son configurables a través de parámetros tales como el número de repeticiones, la velocidad de flexión y extensión de los dedos o el ángulo de la articulación MCP alcanzado, entre otros.

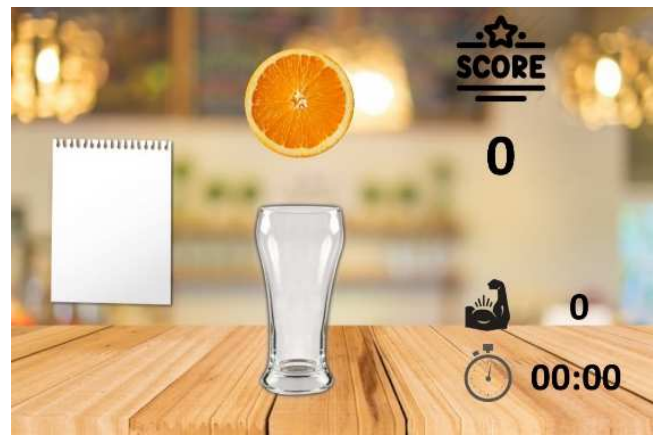

Figura 13: Interfaz de la terapia Orange Juice

Los pacientes que realizan terapias basadas en videojuegos que proporciona una realimentación al paciente sobre el grado de progreso de la rehabilitación, los encuentran menos aburridos que las terapias convencionales y reconocen que al conocer el ritmo de su recuperación, refuerza de manera muy positiva su actitud [16]. Con objeto de aumentar la eficacia de la terapia de rehabilitación, se está desarrollando una nueva aplicación que contenga terapias activas de tipo videojuego

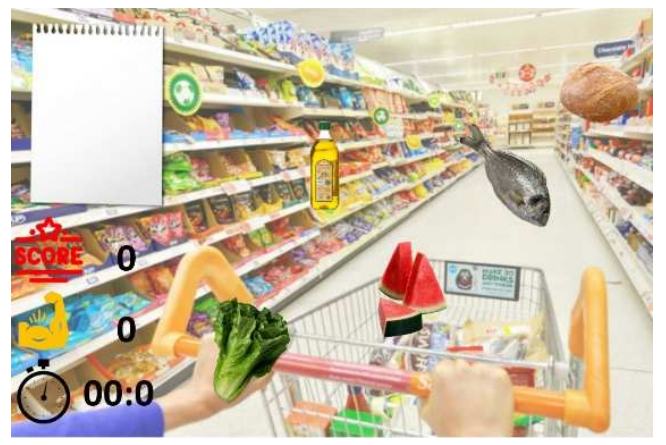

Figura 14: Interfaz de la terapia Supermarket

Las terapias que se están desarrollando tienen como objetivo reestablecer la funcionalidad de apertura y 
cierre de la mano mediante la realización de actividades básicas de la vida cotidiana como exprimir naranjas (Fig. 13) o realizar la compra (Fig 14). Toda la información relacionada con estas nuevas terapias de videojuegos, se guardarán en una base de datos en la "nube", a la que podría acceder el fisioterapeuta para realizar un seguimiento de la evolución del paciente.

\section{CONCLUSIONES}

Se ha presentado el estado actual de desarrollo de Robhand, un nuevo robot para rehabilitación de mano, mediante terapias activas y pasivas, para pacientes que tienen discapacidad neuromotora debido a un ACV. El entorno de rehabilitación integra un exoesqueleto de mano, un sistema de registro, elementos para el procesamiento de señales electromiográficas (sEMG) y un entorno software para la gestión de pacientes, terapias y la realización de tareas de rehabilitación basadas en entornos virtuales. El proyecto Robhand se encuentra en su segundo año de ejecución y, por tanto el desarrollo del sistema está en una etapa intermedia. Los avances realizados en el marco de la realización de este proyecto son una muestra de las posibilidades de la colaboración, en el ámbito de la sanidad, entre organismos públicos de investigación, en este caso un centro universitario, empresas privadas y entidades asistenciales

\section{Agradecimientos}

El trabajo reflejado en está comunicación está siendo realizado gracias a la financiación del Centro para el Desarrollo Tecnológico Industrial (CDTI, referencia IDI 2017 0263) y de la empresa CyL Imas D.

\section{English summary}

\section{ROBHAND, A EXOSQUELETON FOR NEUROMOTOR REHABILITATION OF THE HAND, APPLYING ACTIVE AND PASSIVE THERAPIES}

\author{
Abstract \\ We present the basics and current state of execution \\ of a project that is being carried out through the \\ collaboration of a company $(C y L$ Imas D, \\ Salamanca), two healthcare centers (Hospital \\ Clínico Universitario de Valladolid and Club de \\ Rehabilitación Club de Leones Cruz del Sur, Chile ) \\ and a research center linked to a University (ITAP \\ University Institute, University of Valladolid). The \\ main objective of this project is the creation, \\ development, implementation and clinical trials of a
}

new prototype of active haptic robot for maintenance and neuromotor rehabilitation of the hand in patients with acquired brain damage. The project approach is described, a technical description of the main components of the system is made and its execution status is summarized.

Keywords: Assistential robotics, exoskeletons, neurorehabilitation, Haptic control, sEMG.

\section{Referencias}

[1] Andrianesis, K. \& Tzes, A., (2015) "Development and Control of a Multifunctional Prosthetic Hand with Shape Memory Actuators", J Intell Syst 72(257), pp.257-289.

[2] Fischer, H., Stubblefield, K., Kline, T., Luo, X., Kenyon, R. \& Kamper, D., (2007) "Hand rehabilitation following stroke: a pilot study of assisted finger extension training in a virtual environment", Topics in Stroke Rehabilitation, 14(1):1-12.

[3] Fleischer, C., Wege, A., Kondak, K. \& Hommel, G., (2006) "Application of EMG signals for controlling exoskeleton robots", Biomed Tech, pp. 314-19.

[4] Fraile, J. C., Pérez-Turiel, J., Baeyens, E., Viñas, P., Alonso, R., Cuadrado, A., \& Nieto, F. 2016. E2Rebot: a robotic platform for upper limb rehabilitation in patients with neuromotor disability. Advances in Mechanical Engineering, 8(8), pp. 1-13.

[5] Hidler, J., Nichols, D., Pelliccio, M. \& K. Brady, (2005) "Advances in the understanding and treatment of stroke impairment using robotic devices", Topics Stroke Rehabil., vol. 12 , pp. 22-35.

[6] Hogan, N., Krebs, H., Rohrer, B., Palazzolo, J., Dipietro, L., Fasoli, S., Stein, J., Hughes, R., Frontera, W., Lynch, R. \& Volpe, B., (2006) "Motions or muscles? Some behavioral factors underlying robotic assistance of motor recovery", J. Rehabil. Res. Develop., vol. 45, pp. 605-618.

[7] Kahn, L., Lum, P., Rymer, W. \& Reinkensmeyer, D., (2006) "Robot-assisted movement training for the stroke-impaired arm: Does it matter what the robot does?", J. Rehabil. Res. Develop., vol. 43, pp. 619-630. 
[8] Kai Yap, H., W. K. Ang, B., Hoon Lim, J., C. H. Goh, J. \& Chen-Hua Yeow, R. (2016) “A Fabric-Regulated Soft Robotic Glove with User Intent Detection using EMG and RFID for Hand Assistive Application", 2016 IEEE International Conference on Robotics and Automation (ICRA), pp. 3537-42

[9] Kuriki., H. U., De Azevedo, F. M., Ota Takashashi, L. S., Mello, E. M., Filho, R., \& Alves, N., (2013) "The Relationship Between Electromyography and Muscle Force", EMG Methods for Evaluation Muscle and Nerve Function, Intech, pp. 31-54.

[10] Kwakkel, G., Kollen, B., Van Der Grond, J., \& Prevo, A. J., (2003) "Probability of regaining dexterity in the flaccid upper limb impact of severity of paresis and time since onset in acute stroke”, Stroke, 34(9):2181-2186.

[11] La Viola, J. J. Jr., (1999) "A survey of hand posture and gesture recognition techniques and technology", vol. CS-99-11.

[12] Lucas, L., DiCicco, M. \& Matsuoka, Y., (2004) "An EMG-Controlled Hand Exoskeleton for Natural Pinching", Journal of Robotics and Mechatronics, vol. 16, no. 5, pp. 1-7.

[13] Lucas, L., DiCicco, M. \& Matsuoka, Y., (2011) "An EMG-driven Exoskeleton Hand Robotic Training Device on Chronic Stroke Subjects", 2011 IEEE International Conference on Rehabilitation Robotics.

[14] Polygerinos, P., Galloway, K. C., Sanan, S., Herman M. \& Walsh, C. J. (2015) "EMG Controlled Soft Robotic Glove for Assistance During Activities of Daily Living”, 2015 IEEE International Conference on Rehabilitation Robotics (ICOOR).

[15] Rathore, S., Hinn, A., Cooper, L., Tyroler, H., \& Rosamond, W., (2002) "Characterization of incident stroke signs and symptoms findings from the atherosclerosis risk in communities study", Stroke, 33(11):2718-2721.

[16] Reinkensmeyer, D. J. \& Housman, S. J., (2007) "If can't do it once, why do it a hundred times? Connecting volition to movement success in a virtualenvironments motivates people to exercise the arm after stroke", IEEE, pp. 44-48.
[17] Sandoval-Gonzalez, O. O., Jacinto-Villegas, J., Herrera-Aguilar, I., Portillo-Rodiguez, O., Tripicchio, P., Hernandez-Ramos, M., FloresCuautle, A. \& Avizzano, C., (2016) "Design and Development of a Hand Exoskeleton Robot for Active and Passive Rehabilitation", International Journal of Advanced Robotic Systems 13(66), pp.1-12.

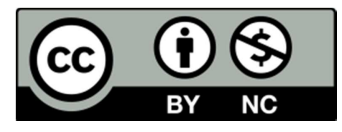

(C) 2018 by the authors. Submitted for possible open access publication under the terms and conditions of the Creative Commons Attribution CC-BY-NC 3.0 license

(https://creativecommons.org/licenses/by-nc/3.0). 